\title{
Landslide susceptibility mapping using an ensemble statistical index (Wi) and adaptive neuro-fuzzy inference system (ANFIS) model at Alborz Mountains (Iran)
}

\begin{abstract}
The main aim of this paper is to develop a new hybrid method to assess landslide susceptibility mapping (LSM) in neighboring provinces of Alborz Mountains in Iran. In the last centuries, this region has experienced a large number of landslides due to its location on earthquake belt, with high precipitation in some parts and having varied topography. Besides, the largest city of Iran (Tehran), a lot of important infrastructures, congested roads and a large population are located in this region. Therefore, determining the spatial outlines of the regions which are prone to future landslides is a critical issue. To reach this goal, the LSM is provided by applying a hybrid model of statistical index (Wi) and adaptive neuro-fuzzy inference system (ANFIS) in a Geographic Information System. In the first step, landslide inventory map was divided into two groups randomly. These groups are training dataset including $70 \%$ recorded landslides and the remaining $30 \%$ was used to test the model output. The first and second groups are used to determine the weights in model and validation of results, respectively. Then, 12 landslide conditioning factors are selected and categorized into two groups which are continuous numerical and nominal. After that, each factor is classified and the weight of each class is determined using Wi. The outputs of Wi and WiANFIS were employed to determine nominal and continuous numerical data, respectively. In the Wi-ANFIS approach, the calculated weights of each class is allocated to the center of each class, and the rest weights of values are determined by ANFIS which is an artificial algorithm using training data (in this paper, the weights were calculated by $\mathrm{Wi}$ ) in terms of predicting and interpolating. The results are evaluated using receiver operation curves including success rate curve and predicted rate curve. The validation results of the proposed hybrid method shows that the area under the curve of success rate curve and predicted rate curve are 0.90 and 0.89 , respectively, which have been improved in comparison with $\mathrm{Wi}$. The results pr oved that the suggested model applied in this study generated reliable LSM which can be applicable for primary land use planning and infrastructure site selection.
\end{abstract}

Keyword: Alborz Mountain; ANFIS; Ensemble; GIS; Iran; Landslide susceptibility 